\title{
Author Correction: Microscale Schottky superlubric generator with high direct-current density and ultralong life
}

Xuanyu Huang (1D, Xiaojian Xiang, Jinhui Nie, Deli Peng, Fuwei Yang (1D, Zhanghui Wu (D), Haiyang Jiang, Zhiping Xu (1) \& Quanshui Zheng (1)

Correction to: Nature Communications https://doi.org/10.1038/s41467-021-22371-1, published online 15 April 2021.

This article contains an error in equation (6) in the original manuscript where one symbol was wrong. The corrected version is:

$$
\begin{gathered}
\overrightarrow{\boldsymbol{J}_{\mathbf{n}}}=-q n \mu_{\mathrm{n}} \nabla V+q D_{\mathrm{n}} \nabla n, \\
\overrightarrow{\boldsymbol{J}_{\mathbf{p}}}=-q p \mu_{\mathrm{p}} \nabla V-q D_{\mathrm{p}} \nabla p, \\
\nabla \cdot \overrightarrow{\mathbf{J}_{\mathbf{n}}}=q \frac{\partial n}{\partial t}, \\
\nabla \cdot \overrightarrow{\vec{J}_{\mathbf{p}}}=-q \frac{\partial p}{\partial t},
\end{gathered}
$$

The original version of this Article (main manuscript and Supplementary Information) contained an error in the spelling of the author Jinhui Nie, which was incorrectly given as Jinghui Nie.

Published online: 14 May 2021

\section{Additional information}

Supplementary information The online version contains supplementary material available at https://doi.org/10.1038/s41467-021-23563-5. 